\title{
The Effect of Green Tea on the Expression of NPC1L1, ABCG5, and ABCG8 in the Intestine of High Fat Diets-induced Rats
}

\author{
Erna Susanti ${ }^{1, *}$, Endang Susilowati ${ }^{2}$ \\ ${ }^{1}$ Academy of Pharmacy and Food Analyst, Putra Indonesia Malang, Jl. Barito No. 5, Lowokwaru, Malang 65123, Indonesia \\ ${ }^{2}$ Academy of Pharmacy, Putra Indonesia Malang, Jl. Barito No. 5, Lowokwaru, Malang 65123, Indonesia \\ *Corresponding author. E-mail: abiyatur@gmail.com
}

Received date: Oct 9, 2020; Revised date: Jan 29, 2021; Accepted date: Feb 5, 2021

\section{Abstract}

2 ACKGROUND: Signaling pathways contributing to cholesterol efflux and inhibitory inflammation in atherosclerosis that has not been explored is the liver X receptor (LXR). Catechin as LXR agonist influences the expression of Niemann-pick C1 like 1 (NPC1L1) protein transporter that triggers the inhibition of cholesterol absorption. This study aimed to examine the effect of Catechins on the expression of intestinal transporters: NPC1L1, ATP-binding cassete-proteins G5 (ABCG5) and G8 (ABCG8).

METHODS: Twenty-five experimental animals were divided into five treatment groups, with 5 rats in each group. The groups were normal diet rats (group 1), high fat diets-induced rats (group 2), high fat diets-induced rats treated with $30 \mathrm{mg} / \mathrm{kg}$ body weight (BW) of Catechins (group 3), high fat diets-induced rats treated with $60 \mathrm{mg} /$ $\mathrm{kg} \mathrm{BW}$ of Catechins (group 4), and high fat diets-induced rats treated with $120 \mathrm{mg} / \mathrm{kg} \mathrm{BW}$ of Catechins (group 5). After one-month, all rats were sacrificed, blood and intestine were collected. Lipid profile were determined enzymatically, mRNA levels were determined by reversetranscriptase polymerase chain reaction (RT-PCR), while the expression of protein transporter were determined by immunohistochemistry.

RESULTS: Catechins treatment decreased the expression of NPC1L1, but increased the expression of ABCG5 and ABG8.

CONCLUSION: Catechins can be developed as a candidate for NPC1L1 inhibitor to mediate the inhibiting absorption of intestinal cholesterol, therefore increasing the inhibitory effect of atherogenesis.

KEYWORDS: ABCG5, ABCG8, aterogenesis, Catechins, green tea, NPC1L1

Indones Biomed J. 2021; 13(2): 147-54

\section{Introduction}

Cardio vascular diseases (CVD) and clinical complications are still occurring despite effective medicines used, hence, complementary therapeutic approaches and new urgent drug developments are to be performed. The main contributor to CVD disease is atherosclerosis. One of the risk factors that cause atherosclerotic prognosis is hypercholesterolemia. A diet high in fat and obesity is one of the triggers of hypercholesterolemia. Hypercholesterolemia can lead to chronic inflammation caused by increased proinflammatory cytokines such as C-reactive protein (CRP) and interleukin-6 (IL-6) that will result in endothelial dysfunction. Endothelial dysfunction plays an important role in the pathogenesis of atherosclerosis is more important because of deregulation of enzymatic activity of endothelial nitric oxide synthase (eNOS) and inactivation of nitric oxide (NO) by oxidative stress. $(1,2)$

Besides causing endothelial dysfunction, the accumulation of toxic metabolites of fat such as fatty acyl CoA, diacylglycerol, ceramide in tissues, including in the arteries will cause insulin resistance because the metabolite will damage the insulin signaling and glucose 
metabolism. This condition will also trigger the acceleration of atherosclerosis. Cholesterol is actually an important part of the body as a major component of biological membranes, is required for the formation of bile salts as well as a precursor of steroid hormones and vitamin D. However, the accumulation of cholesterol in cells, especially in the arterial blood vessels becomes a serious problem that triggers atherosclerosis. Therefore, how to efflux of cholesterol from cells with high cholesterol to be important step for the prevention of atherosclerosis.(3)

One of the signaling pathways that contribute to cholesterol efflux and inhibitory inflammation in atherosclerosis that has not been explored is the liver $\mathrm{X}$ receptor (LXR). The lipid homeostasis process plays a major role in the LXR signaling pathway. LXR regulates transcription factors that play a role in lipid homeostasis.(4)

The decrease in the expression of Niemann-pick C1 like 1 (NPC1L1) on the enteric apical membrane has an effect on the inhibition of cholesterol absorption. LXR agonists play a role in decreasing NPC1L1 expression. NPC1L1 is a protein in the apical of enterocytes that play an important role in the absorption of intestinal cholesterol. Cholesterol was esterified in enterocytes by acyl CoA cholesterol acyltransferase-2 (ACAT-2) and forming Chylomicrons. Some cholesterol is transported through ATP-binding cassette transporter A1 (ABCA1) to Apolipoprotein A1 (ApoA1), the main process of high-density lipoprotein (HDL) formation. Some intracellular cholesterol is removed from the enterocytes into the lumen intestinal through ATP-binding cassete-proteins G5 (ABCG5) / ATPbinding cassete-proteins G8 (ABCG8), the heterodimer located in the apical membrane.(5) Cholesterol dietary and biliary cholesterol are synthesized in the gut by HMG CoA transported across the cell membrane by NPC1L1 and together with triglycerides, phospholipids, ApoB48 were synthesized into triglyceride-rich chylomicrons under the influence microsomal triglyceride transport protein (MTP). Some of the absorbed cholesterol is excreted in the gut with the help of ABCG5/ABCG8. It is expected that the scientific evidence of the effects of catechins through the mechanism of inhibition absorption of intestinal cholesterol can increase the potential effect of Catechins for the treatment of atherosclerosis and cardiovascular diseases generally.

To prove the effect of Catechins on inhibition of intestinal cholesterol absorption, an in vivo model is required. This effect is proved through the inhibition of NPC1L1 expression and the increase of ABCG5 and ABCG8 expression. This inhibitory effect tests were carried out by measuring the NPC1L1, ABCG5 and ABCG8 expressions.
The aim of this study was to prove the inhibitory effect of Catechins on intestinal cholesterol absorption through in vivo studies.

\section{Methods}

\section{Animal Care and Experimental Diets}

In this research, 25 experimental male Wistar rats aged 5 weeks were divided into 5 different treatment groups, with 5 rats in each group. Treatment duration was 4 weeks and the rats was acclimatized for 1 week previously.

After acclimatization for 7 days with a normal diet, atherogenic diets was prepared according to previous study.(6) Table 1 showed the composition of the experimental atherogenic diet. Rats were divided randomly into five groups that were: (1) normal diet rats; (2) high fat diet-induced rats; (3) high fat diet-induced rats treated with Catechins $30 \mathrm{mg} / \mathrm{kg}$ body weight (BW); (4) high fat diet-induced rats treated with Catechins $60 \mathrm{mg} / \mathrm{kg} \mathrm{BW}$; and (5) high fat diet-induced rats treated with Catechins $120 \mathrm{mg} /$ $\mathrm{kg} \mathrm{BW}$. All of groups were given free access to water and the experimental diet. After treatment, rats were weighed and sacrificed. Blood was collected from the descending aorta, and serum was prepared by centrifugation at 1500 rpm for 15 minutes then stored at $80^{\circ} \mathrm{C}$ until analysis. Intestine tissue was rapidly removed, rinsed and kept at $80^{\circ} \mathrm{C}$.

The experimental protocol was reviewed and approved by the Ethics Committee of Faculty of Medicine, Universitas Brawijaya (207/EC/KEPK/06/2017).

\section{Determination of Lipid Profiles}

Total cholesterol (TC), triglycerides (TG) and HDLcholesterol (HDL-C) were measured enzymatically by colorimetry. TG level was determined by cholesterol oxidase-peroxidase aminoantypirin

(CHOD-PAP)

Table1. Composition of normal and atherogenic diets.(6)

\begin{tabular}{lcc}
\hline \multicolumn{1}{c}{ Component } & Normal Diet & Atherogenic Diet \\
\hline PARS/chicken feed & $225 \mathrm{~g}$ & $200 \mathrm{~g}$ \\
Wheat flour & $100 \mathrm{~g}$ & $100 \mathrm{~g}$ \\
Cholesterol & - & $8 \mathrm{~g}$ \\
Cholic acid & - & $0.8 \mathrm{~g}$ \\
Pig oil & - & $10 \mathrm{~mL}$ \\
Distilled water & $100 \mathrm{~mL}$ & $81.2 \mathrm{~mL}$ \\
\hline
\end{tabular}

PARS/chicken feed consist of: water, proteins, fats, fiber, ash, $\mathrm{Ca}$, phosphorus, antibiotics, coccidiostat. 
enzymatic photometric test. Atherogenic index was calculated according to the following equation: atherogenic index $=$ TC/HDL-C.(7)

\section{Immunohistochemistry}

The immune staining for NPC1L1, ABCG5, ABCG8 was performed using Polyclonal Antibody (Bioss Antibodies, Woburn, MA, USA). Positive cells expression were identified by a brown staining, while the expression was demonstrated on brush border membrane from jejunum. In order to minimize the misinterpretation of immunohistochemical results, two different observers were carried out by using five slides taken randomly for each experimental animal. Each tissue sections were examined under a light microscope.

Expression of NPC1L1 was evaluated by the scoring system of IRS classification.(8) The fraction of positive cells were classified $(0 \%=0 ;<10 \%=1 ; 10$ to $50 \%=2$; 51 to $80 \%=3 ;>80 \%=4)$ and the staining intensity were classified (negative $=0$; weak $=1$; moderate $=2$; strong $=$ 3). Immunoreactive score (IRS) with points $0-12$ obtained from multiplication of fraction of positive cells and staining intensity classified into 4-point-IRS-classification ( 0 to $1=$ $0 ; 2$ to $3=1 ; 4$ to $8=2$; and 9 to $12=3$ ). Significantly of difference among groups was analyzed by the SPSS software. The accepted level of significance was $p<0.05$.

\section{Real-time (RT)-PCR mRNA Analysis}

RNA was isolated from $30 \mathrm{mg}$ frozen intestinal tissue with the RNeasy-mini kit (Qiagen, Hilden, Germany) according to the manufacturer's guidelines. Prior to the RTPCR analysis, the RNA concentration was determined at a wavelength of $260 \mathrm{~nm}$. The absorbance ratio of A260/A280 should be in the range 1.8 to 2.2. RT-PCR was performed using the SYBR Green PCR Master Mix on a GeneAmp 5700 Sequence Detection System (Applied Biosystems, Foster City, CA, USA). The results of the RT-PCR obtained were compared with $\beta$-actin expression as a housekeeping gene.(9)

\section{Statistical Analysis}

The research data were in the form of qualitative data and quantitative data. Quantitative data are presented as mean \pm SD. Statistical analysis used ANOVA followed by the Tukey test to prove whether there was a significant difference or not from the research results. The level of significance was at $p<0.05$. SPSS software (IBM Coorporation, Armonk, NY, USA) was used for statistical analysis.

\section{Results}

\section{Effect of Catechins Treatment on Blood Lipid Levels}

The results showed that Catechins significantly reduced TC, TG, HDL-C in group of high fat diets-induced rats (Table 2). Atherogenic index was markedly reduced respectively in Catechins treatment group compared with high fat dietsinduced rats group. The high levels of TC, TG, and HDL-C were decreased significantly after one month of Catechins treatment. Total cholesterol of serum from rats that had high fat diets was higher than rats that had normal diet. The TC of serum from dose I and dose II of Catechins groups was lower than high fat diet-induced rats.

\section{mRNA NPC1L1, ABCG5, ABCG8 Expression Level}

The quantitative measurement of mRNA was based on the standard of housekeeping gene $\beta$-actin with various concentrations. To homogenize the number of samples to be analyzed was determined by levels of RNA that was isolated by using nanodrop with $100 \mathrm{ng}$ per sample. Primers and result of quantification of mRNA NPC1L1, ABCG5, ABCG8 were showed at Table 3 and Figure 1.

\section{Immunohistochemical NPC1L1 Expression in Jejunum}

One of the transporter proteins that mediate cholesterol uptake on the plasma membrane is NPC1L1. The NPC1L1 immune staining results showed positive immunoreaction (brown color) on brush border membrane from jejunum (Figure 2).

Table 2. Lipid profiles in different studied groups ( $n=5$ in each group).

\begin{tabular}{lccccc}
\hline Biochemical Factors & Control (-) & Control $(+)$ & $\begin{array}{c}\text { Dose I } \\
\mathbf{( 3 0} \mathbf{~ m g} / \mathbf{k g ~ B W})\end{array}$ & $\begin{array}{c}\text { Dose II } \\
\mathbf{( 6 0} \mathbf{~ m g} / \mathbf{k g ~ B W})\end{array}$ & $\begin{array}{c}\text { Dose III } \\
(\mathbf{1 2 0} \mathbf{~ m g} / \mathbf{k g ~ B W})\end{array}$ \\
\hline $\mathrm{TC}(\mathrm{mg} / \mathrm{dL})$ & $54.80 \pm 8.84$ & $85.25 \pm 26.11$ & $71.75 \pm 6.95$ & $80.67 \pm 16.20$ & $85.67 \pm 5.86$ \\
$\mathrm{TG}(\mathrm{mg} / \mathrm{dL})$ & $70.75 \pm 16.72$ & $86.00 \pm 14.89$ & $57.00 \pm 17.06$ & $44.60 \pm 9.50$ & $43.75 \pm 9.63$ \\
HDL-C (mg/dL) & $43.20 \pm 10.55$ & $61.00 \pm 23.14$ & $58.30 \pm 20.01$ & $35.75 \pm 3.77$ & $42.40 \pm 8.29$ \\
Atherogenic index & $1.19 \pm 0.02$ & $1.63 \pm 0.19$ & $1.02 \pm 0.45$ & $1.18 \pm 0.01$ & $1.48 \pm 0.25$ \\
\hline
\end{tabular}

Data represented as mean $\pm \mathrm{SD}$. 
Tabel 3. Primers for ABCG5, ABCG8, NPC1L1 and $\beta$ Actin used in this study.

\begin{tabular}{|c|c|}
\hline Genes & Primer \\
\hline \multirow{2}{*}{ ABCG5 } & Forward : TGCCTTTCTGAGTCCAGAG \\
\hline & Reverse : GTGCTCTTTCAATGTTCTCCAG \\
\hline \multirow{2}{*}{ ABCG8 } & Forward :ATGAGCTGGAAGACGGGCTG \\
\hline & Reverse : GCCAGTGAGAGCAAGGCTGA \\
\hline \multirow{2}{*}{ NPC1L1 } & Forward: GCTCTCTCCGCAAGATATACACTC CC \\
\hline & Reverse: GAGGATGCAGCAATAGCCACATAAGAC \\
\hline \multirow{2}{*}{$\beta$-Actin } & Forward: TGGAATCCTGTGGCATCCATGAAAC \\
\hline & Reverse : TAAAACGGAGCTCAGTAACAGTCCG \\
\hline
\end{tabular}

Compared to high fat diet-induced rats, Catechins increased the expression of ABCG5 especially in the dose of $60 \mathrm{mg} / \mathrm{kg}$ BW of Catechins. All of dose Catechins showed to increase the expression of ABCG5 (Figure 3). The intensity of brown colour increased at all of doses Catechins.

Figure 4 showed there were increasing intensity of brown colour especially in the dose of $60 \mathrm{mg} / \mathrm{kg} \mathrm{BW}$ of Catechins. All of dose Catechins increase ABCG8 expression in rats jejunum. ABCG5 and ABCG8 are the two proteins that form heterodimers in the endoplasmic reticulum and then traffic to the apical membrane, where they transport neutral sterols into bile or into the gut lumen.

\section{Discussion}

Epigallocatechin gallate (EGCG), which is one of the active compounds of catechins from green tea, plays a role in micelle formation and the lipid hydrolysis process, which is the main step in the transfer ofhydrolyzed lipids for uptake via enterocytes. The in vitro studies have shown that catechins from Green Tea affect the hydrolysis and stabilization of luminal lipid micellar. Research shows EGCG effectively precipitate cholesterol from bile acid micelles but did not significantly affect the solubility of micellar fatty acids and monoacyl glycerol from hydrolysis of triglycerides in the intestinal lumen by pancreatic lipase.(10)

Statistical analysis showed that there was an increase in ABCG5 and ABCG8 mRNAs in all three doses of Catechins while a significant increase occurred at Catechins $60 \mathrm{mg} / \mathrm{kg}$ BW dose compared to the group given only a high-fat diet with a value of $p<0.05$. Activation of LXR signaling as a result of cholesterol diet will lead to reduced cholesterol absorption and increased excretion of bile were mediated by ABCG5 and ABCG8. Mice lacking ABCG5 and ABCG8 did not accumulate excessive levels of plasma and tissue cholesterol. ABCG5 and ABCG8 are transporter proteins required for the elimination of cholesterol and
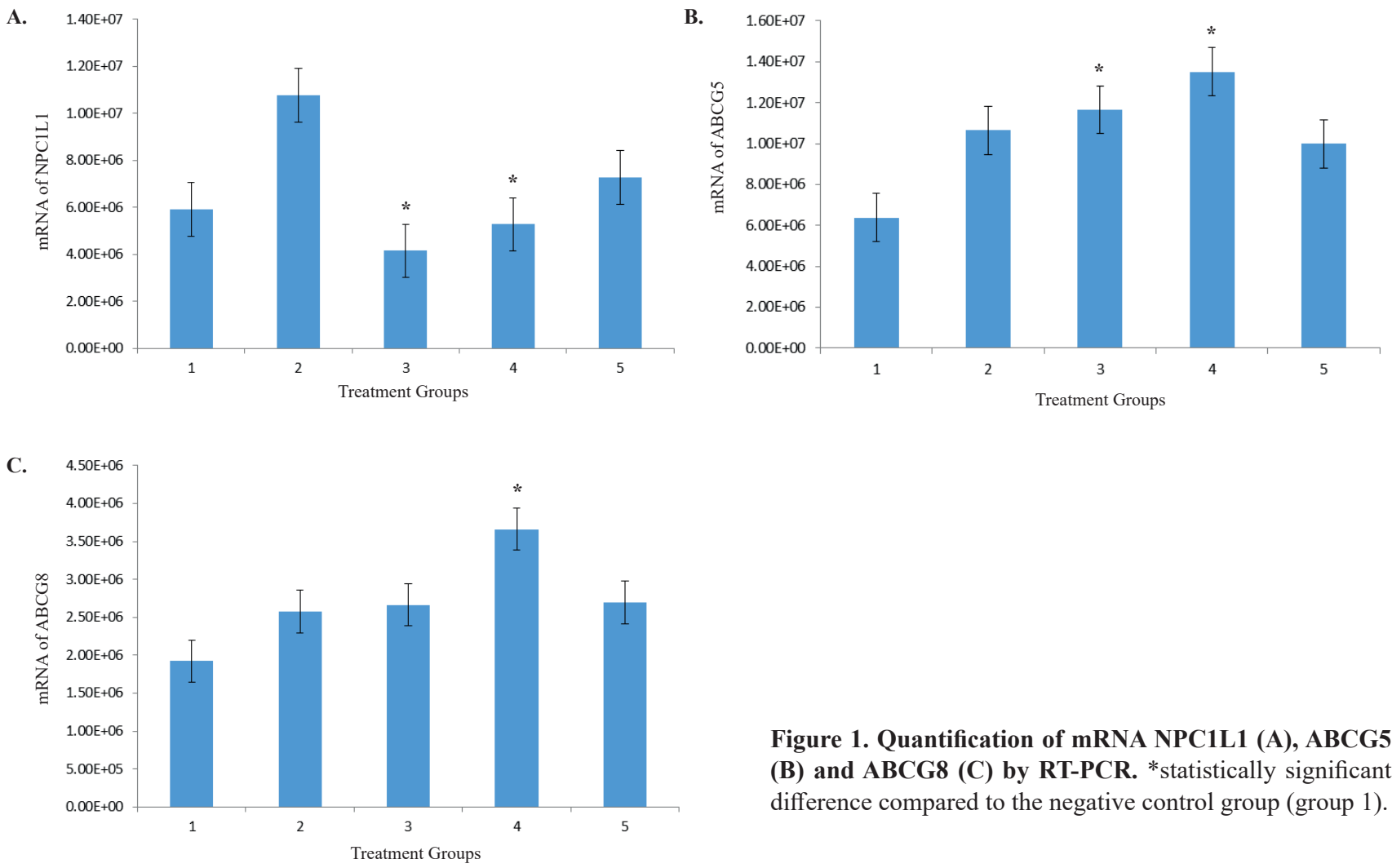

Figure 1. Quantification of mRNA NPC1L1 (A), ABCG5 (B) and ABCG8 (C) by RT-PCR. *statistically significant difference compared to the negative control group (group 1). 

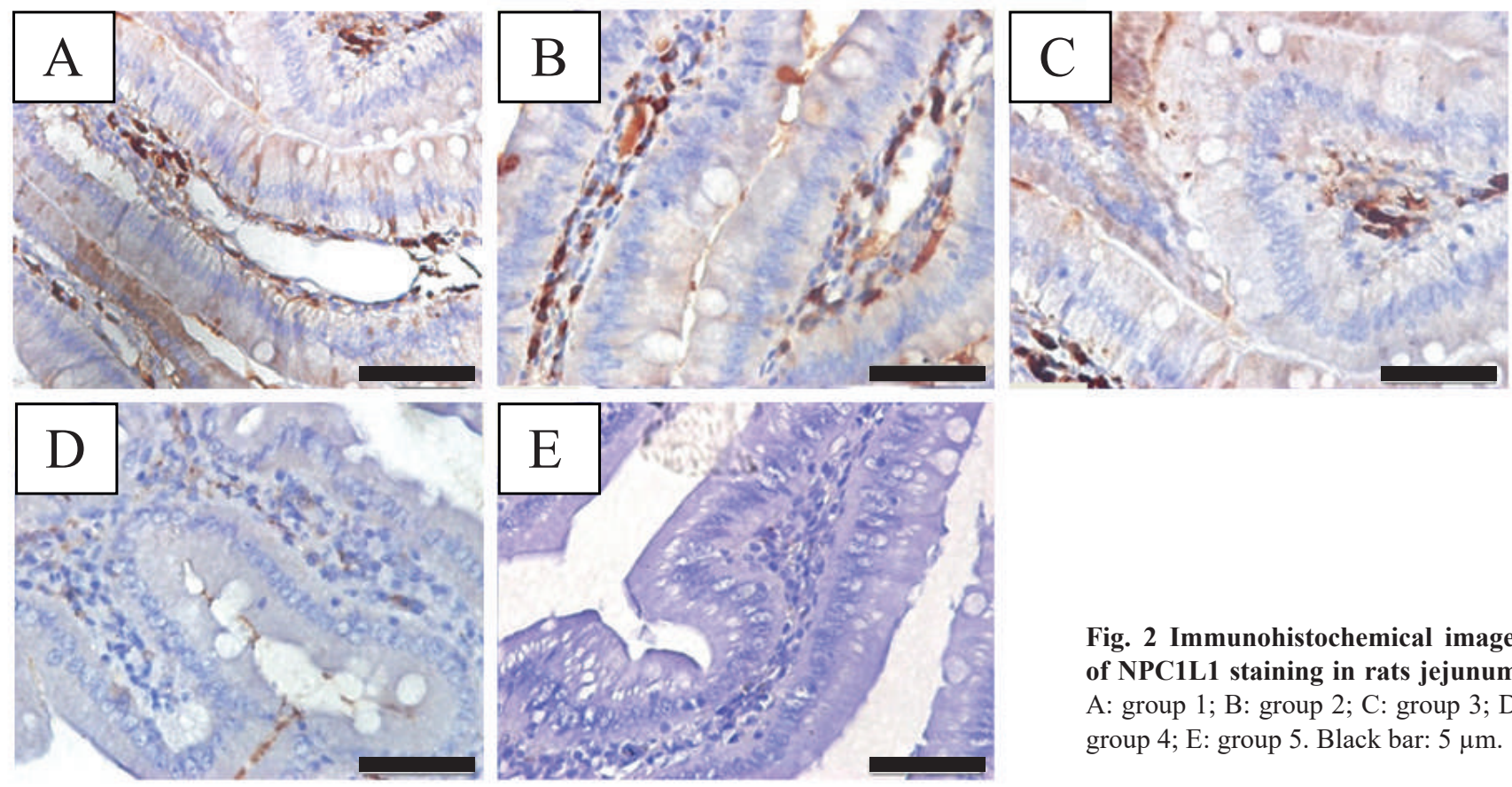

Fig. 2 Immunohistochemical images of NPC1L1 staining in rats jejunum. A: group 1; B: group 2; C: group 3; D: group 4; E: group 5. Black bar: $5 \mu \mathrm{m}$.

reducing absorption of cholesterol mediated by LXR. Although upregulation of ABCG5 and ABCG8 mRNA had no significant effect on the increase biliary output of cholesteol. Mice lacking LXR- $\alpha$ caused failure to upregulate ABCG5 and ABCG8 in response to cholesterol feeding but cholesterol excretion was the same as wild type mice which showed four to five fold 5 increase in mRNA.(11) Hepatocyte nuclear factor (HNF) 4- $\alpha$ bound to GATA-binding protein 4 (GATA4) and GATA6 shows synergistic activity to increase the expression of ABCG5 and ABCG8. Furthermore, this study shows for the first time that Catechins up-regulates the expression of these ABC transporters at the mRNA level. LXR $\alpha$ by controlling expression of a number of genes, such as NPC1L1 protein, heterodimers of ABCG5 and ABCG8 reduces intestinal cholesterol absorption and therefore regulated plasma cholesterol.(4)

In this experiment mRNA of NPC1L1 was significantly reduced in Catechins-treated group compared with the high-fat diet group. Cholesterol absorption in NPC1L1 null mouse showed nearly $90 \%$ reduction. NPC1L1 inhibition
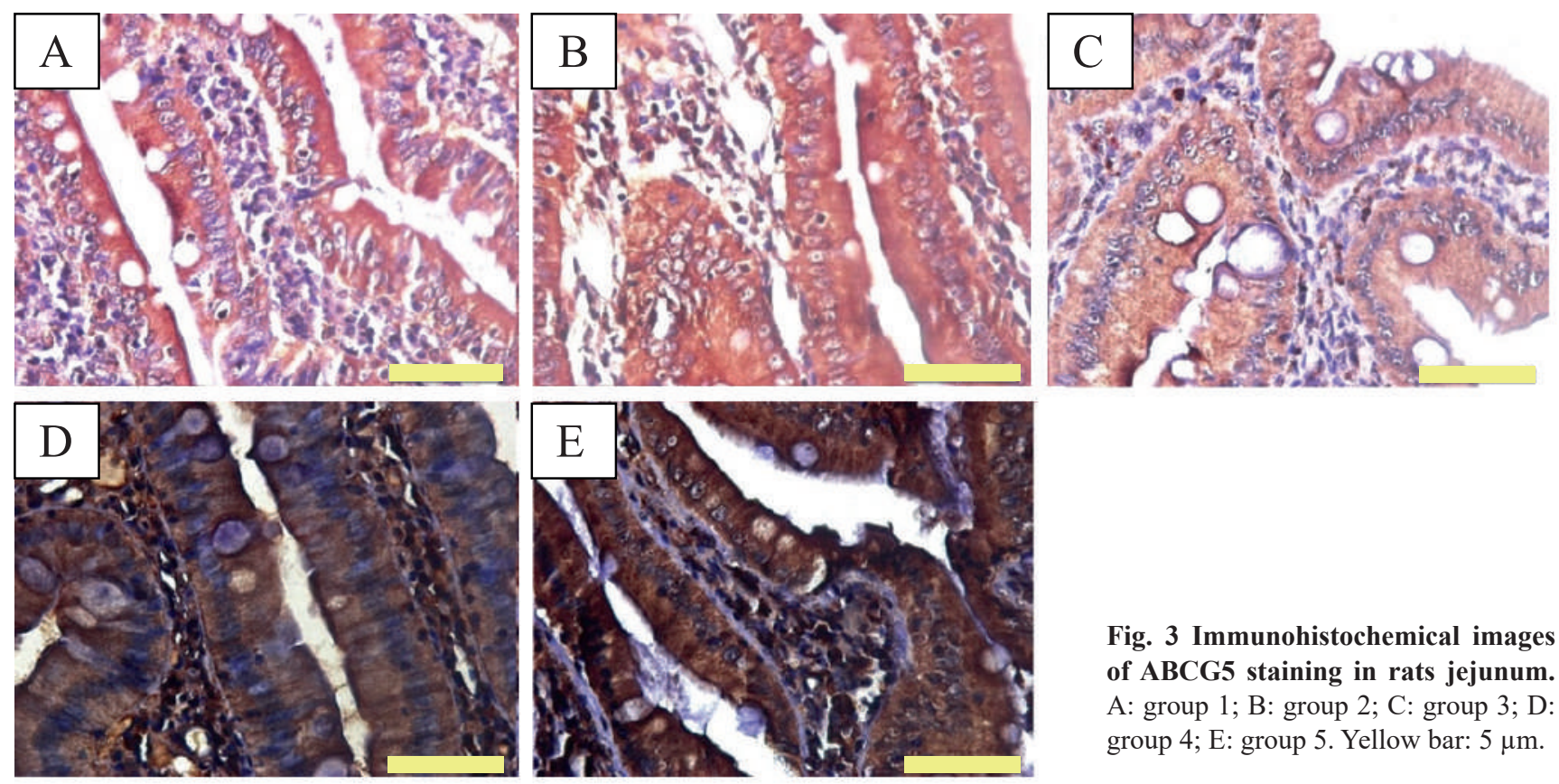

Fig. 3 Immunohistochemical images of ABCG5 staining in rats jejunum. A: group 1; B: group 2; C: group 3; D: group 4; E: group 5. Yellow bar: $5 \mu \mathrm{m}$. 

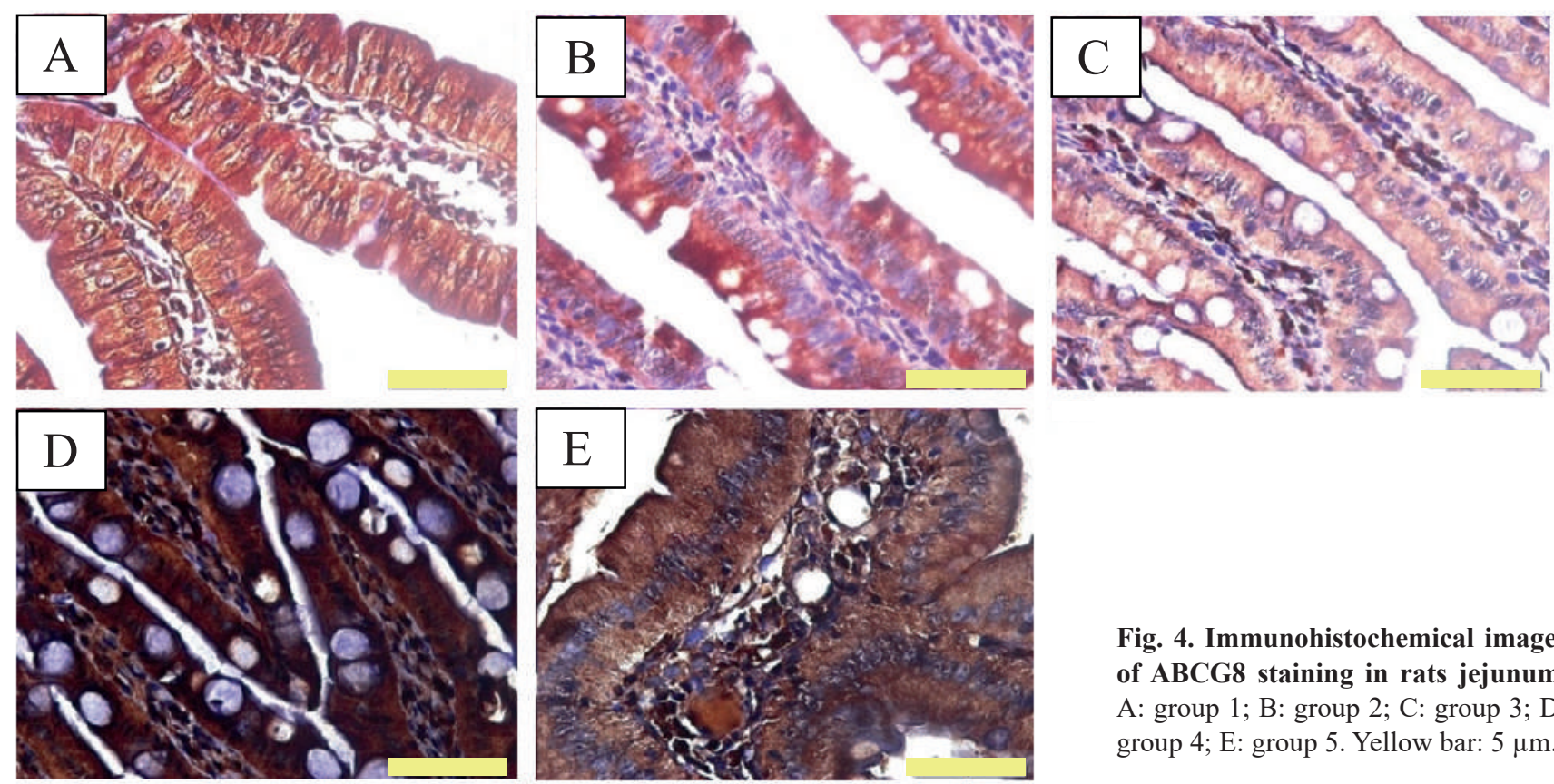

Fig. 4. Immunohistochemical images of ABCG8 staining in rats jejunum. A: group 1; B: group 2; C: group 3; D: group 4; E: group 5. Yellow bar: $5 \mu \mathrm{m}$.

should have useful effects on atherosclerosis. Treatment with Catechins significantly reduces mRNA NPC1L1, reduces cholesterol absorption, lowers total cholesterol, and also may inhibit the development and progression of atherosclerosis in high fat diet rats.

The immunohistochemistry results showed that administration of Catechins decreased NPC1L1 expression. Decreasing of NPC1L1 expresssion was shown by decreasing brown staining from immunohistochemical result. As much as $0.5 \%$ green tea moderately increased intestinal NPC1L1 mRNA that plays a role in the uptake of intestinal cholesterol.(10) Other studies have shown that EGCG and caffeine infundation either single or in combination significantly inhibits intestinal lipid absorption. Curcumin significantly reduced the mRNA and expression of NPC1L1 protein in intestinal Caco-2 monolayers. Analysis of mRNA and protein expression was performed with Western blot and qPCR.(10)

The abundance expression of ABCG5 and ABCG8 but not mRNA is reduced in mice lacking a functional leptin axis. The defect of the leptin axis was associated with reduced levels of immunoreactive ABCG5 and ABCG8, transpoter sterols. The reduction in immunoreactive levels is not related to a decrease in the level of mRNA encoding the gene. Caloric restriction can restore levels of the sterol transporter ABCG5 and ABCG8. Administration of Tauro Urso Deoxy Cholate (TUDCA) can restore immunoreactive G5 and G8 in db/db mice, but also increase ABCG5/ABCG8 in wildtype. TUDCA caused an increase in ABCG5/ ABCG8 abundance which was associated by an increase in biliary cholesterol concentration. The leptin hormone plays a role in the availability of ABCG5 and ABCG. Replacement leptin can also restore ABCG5 and ABCG8. TUDCA plays a role in the availability of ABCG5 and ABCG8 levels through the endoplasmic reticulum stress and the role of Unfolded Protein Response (UPR). Polymorphisms in ABCG5 and ABCG8 are associated with responses to cholesterol metabolism following weight loss.(11,12)

ABCG5 andABCG8 and the NPC1L1 protein play a critical role in intestinal cholesterol absorption and secretion of cholesterol back into the intestinal lumen for fecal removal. Treatment of mice by LXR agonist increased excretion of sterols, thus reducing cholesterol absorption This effect is mediated through ABCG5 and ABCG8. Activation of LXR, increased expression of both ABCG5 and $\mathrm{ABCG} 8$, which transferred absorbed cholesterol back to the intestinal lumen.(13)

Cholesterol homeostasis is determined by endogenous synthesis, intestinal absorption and hepatic excretion. Increased levels of cholesterol in the blood are a risk factor for atherosclerosis, which leads to cardiovascular diseases. NPC1L1 whose expression is present on the enterocyte membrane and membrane of hepatocytes acts as a sterol transporter that mediates absorption of intestinal cholesterol. Ezetimibe is a potent drug as an inhibitor of intestinal cholesterol absorption acting by means of inhibiting NPC1L1. This drug is widely used in the treatment of hypercholesterolemia.

The expression of NPC1L1 in humans and in animals is different. NPC1L1 expression in humans is in the small 


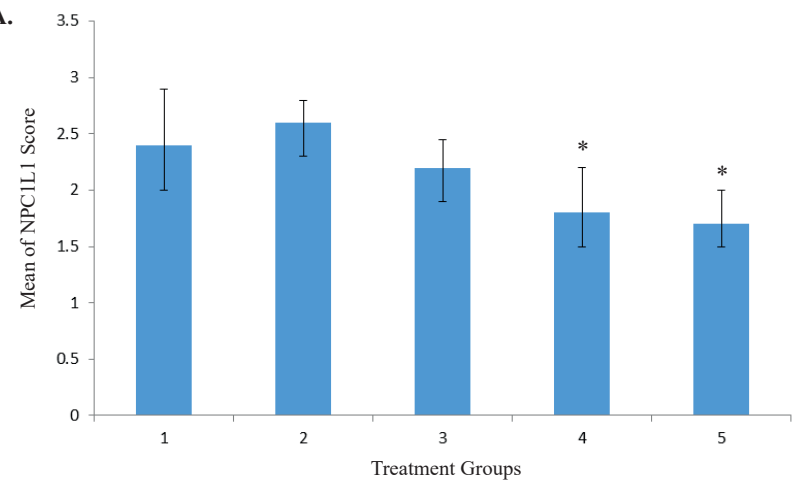

C.

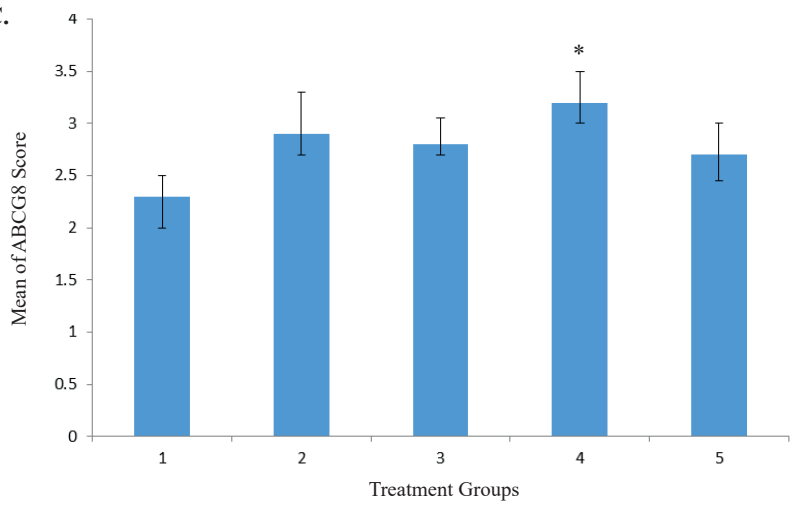

intestine and liver. NPC1L1 is regulated by sterol regulatory element-binding protein 2 (SREB2) transcription factor, which increases the expression of cholesterol synthesis gene when cellular cholesterol levels are low. Mice lacking ACAT2 or phospholipid transfer protein (PLTP) accumulate unesterified cholesterol in enterocytes and simultaneously show reduced intestinal NPC1L1 mRNA levels.(14) In small intestine, unesterified cholesterol will enter the endoplasmic reticulum and undergo esterification by ACAT2 before the process is packed into the cylomicron. Research shows NPC1L1 knockout mice and ezetimibetreated mice show similarly reduced intestinal cholesterol absorption. This research shows that NPC1L1 is the target of ezetimibe. Ezetimibe binds to a single site on intestinal brush border membrane vesicles and to cells overexpressing NPC1L1 and this binding is absent in intestinal brush border membrane vesicles isolated from NPC1L1-null mice.(15-17)

\section{Conclusion}

In conclusion, Catechins significantly reduced lipid profile and atherogenic index, as compared with the rat induced high fat diet groups. Catechins treatment also led to downregulation of NPC1L1 mRNA and up-regulation of ABCG5

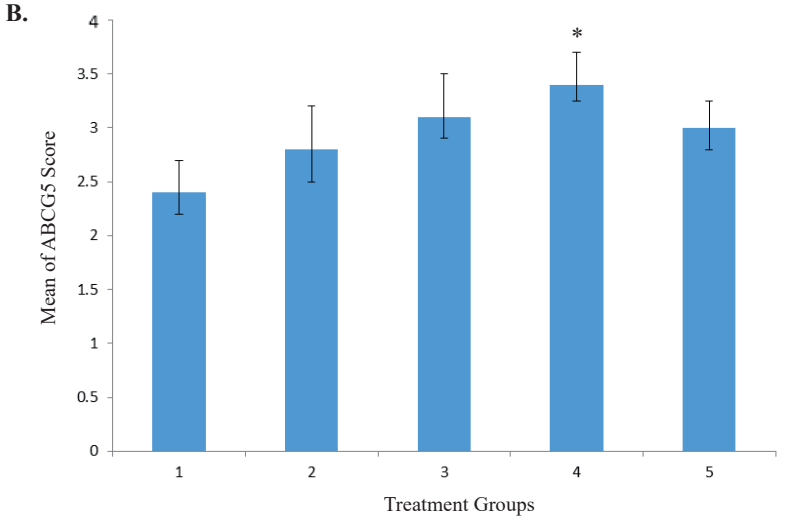

Figure 5. Scores mean of NPC1L1 (A), ABCG5 (B), ABCG8 (C) expression in rats jejunum. Data are expressed as mean $\pm \mathrm{SD}$ of each group. *statistically significant difference compare to negative control group (group 1).

and ABCG8 mRNAs in the intestine of rats. These results provide that Catechins decreases the expression of NPC1L1, so it can be developed as a candidate inhibitor of NPC1L1 inhibiting absorption of cholesterol intestinal. Catechins increase the expression of ABCG5 and ABCG8 thereby increasing cholesterol intake into the lumen intestinal. This research shows potential catechins as inhibitors of atherogenesis by activation of LXR signaling by decreasing NPC1L1 expression and increasing ABCG5 and ABCG8 expression.

\section{Acknowledgements}

The authors thank the Directorate General of Research and Development Strengthening, Ministry of Research, Technology and Higher Education of the Republic of Indonesia for the Research Grant.

\section{Authors Contribution}

The first author implementated the research in general, including data collecting and analysis, also preparing the manuscripts. While the second author conducted statistical analysis and assisted in the RT-PCR analysis. 


\section{References}

1. Yang Z, Ming MF. Recent advances in understanding endothelial dysfunction in atherosclerosis. Clin Med Res. 2006; 4: 53-65.

2. Velde AV. Reverse choleterol transport: from classical view to new insight. World J Gastroenterol. 2010; 16: 5908-15.

3. Meiliana A, Wijaya A. Inflammation and atherosclerosis. Indones Biomed J. 2012; 4: 73-83.

4. Calkin A, Tontonoz P. LXR signaling pathway and atherosclerosis. Arterioscler Thromb Vasc Biol. 2010; 30: 1513-8.

5. Wang X, Collins HL, Ranalletta M, Fuki IV, Billheimer JT, Tall AR, et al. Macrophage ABCA1 and ABCG1, but not SR-BI, promote macrophage reverse cholesterol transport in vivo. J Clin Invest. 2007; 117; 2216-24.

6. Murwani S, Ali M, Muliartha K. Diet aterogenik pada tikus putih (Rattus novergicus strain wistar) sebagai model hewan aterosklerosis. Jurnal Kedokteran Brawijaya. 2006; 22: 6-9.

7. Artiss JD, Zak B. Measurement of cholesterol concentration. In: Handbook of Lipoprotein Testing. Washington: AACC Press, 1997. p.99-114.

8. Kaemmerer D, Peter L, Lupp A, Schulz S, Sanger J, Baum R, et al. Comparing of IRS and Her2 as immunohistochemical scoring schemes in gastroenteropancreatic neuroendocrine tumors. Int $\mathrm{J}$ Clin Exp Pathol. 2012; 5: 187-94.

9. Cimmino G, Ibanez B, Vilahur G, Speidl WS, Fuster V, Badimon L, et al. Up-regulation of reverse cholesterol transport key players and rescue from global inflammation by ApoA-I (Milano). J Cell Mol Med. 2009; 13: 3226-35.
10. Shrestha S, Ehlers SJ, Lee J, Fernandez M, Koo S. Dietary green tea extract lowers plasma and hepatic triglycerides and decreases the expression of sterol regulatory element-binding protein-1c mRNA and its responsive genes in fructose-fed, ovariectomized rats. J Nutr. 2009; 139: 640-5.

11. Berge KE, Tian H, Graf GA, Yu L, Grishin NV, Schultz J, et al. Accumulation of dietary cholesterol in sitosterolemia caused by mutations in adjacent ABC transporters. Science. 2000; 290: 1771-5.

12. Wang J, Mitsche MA, Lütjohann D, Cohen JC, Xie XS, Hobbs HH. Relative roles of ABCG5/ABCG8 in liver and intestine. J Lipid Res. 2015; 56: 319-30.

13. Mohammadi A, Mirzaei F, Moradi MN, Jamshidi M, Yari R, Ghiasvand $\mathrm{T}$, et al. Effect of flaxseed on serum lipid profile and expression of NPC1L1, ABCG5 and ABCG8 genes in the intestine of diabetic rat. Avicenna J Med Biochem. 2013; 1: 1-6.

14. Jia L, Better JL, Yu L. Niemann-pick C1-like 1 (NPC1L1) protein in intestinal and hepatic cholesterol transport. Annu Rev Physiol. 2011; 73: 239-59.

15. Garcia-Calvo M, Lisnock J, Bull HG, Hawes BE, Burnett DA, Braun MP, et al. The target of ezetimibe is Niemann-pick C1-like 1 (NPC1L1). Proc Natl Acad Sci. 2005; 102: 8132-7.

16. Nakano T, Inoue I, Takenaka Y, Ono H, Katayama S, Awata T, et al. Ezetimibe promotes brush border membrane-to-lumen cholesterol efflux in the small intestine. PLoS One. 2016; 11: 1-27.

17. Braun A, Yesilaltay A, Acton S, Broschat KO, Krul ES, Napawan N, et al. Inhibition of intestinal absorption of cholesterol by ezetimibe or bile acids by SC-435 alters lipoprotein metabolism and extends the lifespan of SR-BI/apoE double knockout mice. Atherosclerosis. 2008; 198: 77-84. 